\title{
PW03-007 - NLRP3 genetic variants in Schnitzler's syndrome
}

\author{
HD De Koning ${ }^{1 *}$, J Schalkwijk', JW van der Meer ${ }^{2}$, PL Zeeuwen ${ }^{1}, K^{1}$ Neveling ${ }^{3}, M^{2}$ van Gijn ${ }^{4}$, A Simon ${ }^{2}$ \\ From 7th Congress of International Society of Systemic Auto-Inflammatory Diseases (ISSAID) \\ Lausanne, Switerland. 22-26 May 2013
}

\section{Introduction}

Schnitzler's syndrome (SchS) is an autoinflammatory disorder, characterized by chronic urticaria, fever, gammopathy and bone pain. The etiology is unknown, but interleukin-1 (IL-1) inhibition is very effective, like in the cryopyrin associated periodic syndrome (CAPS), that is caused by activating NLRP3 mutations. Previously, a V198M mutation in NLRP3 was reported in one patient with SchS, but this is a prevalent variation in the general healthy population.

\section{Objectives}

To study presence and significance of NLRP3 genetic variants in SchS.

\section{Methods}

We performed exome screening on peripheral bloodderived DNA of three patients with SchS, and Sanger sequencing of NLRP3 on peripheral blood-derived DNA of 9 patients with SchS. Patients were further clinically characterized and cytokine stimulation studies with peripheral blood mononuclear cells (PBMCs) were performed.

\section{Results}

We found NLRP3 genetic variants in two patients. Exome screening revealed the known pathogenic CAPScausing NLRP3 c.1575C > G p.(P525L) mutation in one patient. Confirmation by Sanger sequencing on peripheral blood only showed a small aberrant peak at the corresponding location. In another patient, we found a hitherto unknown NLRP3 variant c.1303A>G p.(K435E), of which the pathogenicity still needs to be determined. None of the patients had clinically affected family members. No V198M mutation in NLRP3 was detected in our population of SchS.

The two patients with NLRP3 variants fulfilled the criteria for SchS, and had the most severe clinical phenotype of the group. Also, both patients had IgG instead of IgM gammopathy, and both patients had the highest production of IL-1 and IL-6 upon stimulation of PBMCs with LPS.

\section{Conclusion}

In seven of nine patients with SchS, no NLRP3 mutations were found. Two patients with IgG-type SchS with a severe phenotype carried a genetic variation in the NLRP3 gene: in one, the novel variant K435E, and in the other one a known mutation P525L that was described in severe CAPS patients.

We hypothesize that somatic mosaicism or a less pathogenic effect of the novel mutation may explain the late onset of symptoms.

\section{Disclosure of interest}

None declared.

\section{Authors' details}

'Dermatology, Radboud University Nijmegen Medical Centre, Nijmegen, Netherlands. ${ }^{2}$ Internal Medicine, Radboud University Nijmegen Medical Centre, Nijmegen, Netherlands. ${ }^{3}$ Genetics, Radboud University Nijmegen Medical Centre, Nijmegen, Netherlands. ${ }^{4}$ Genetics, UMC Utrecht, Utrecht, Netherlands.

Published: 8 November 2013

doi:10.1186/1546-0096-11-S1-A233

Cite this article as: De Koning et al.: PW03-007 - NLRP3 genetic variants in Schnitzler's syndrome. Pediatric Rheumatology 2013 11(Suppl 1):A233.

'Dermatology, Radboud University Nijmegen Medical Centre, Nijmegen,

Netherlands

Full list of author information is available at the end of the article

(c) 2013 De Koning et al; licensee BioMed Central Ltd. This is an Open Access article distributed under the terms of the Creative Commons Attribution License (http://creativecommons.org/licenses/by/2.0), which permits unrestricted use, distribution, and reproduction in any medium, provided the original work is properly cited. 